\title{
Opportunities and Challenges of Machine Vision for Navigation and Obstacle Detection in Autonomous Ground Vehicles
}

\author{
G.D. Suriya Prasath, M.K. Rahgul Poopathi, Swaroop Balaji, H. Sridhar, S. Santhosh
}

\begin{abstract}
Autonomous vehicles are the future of transport and also it is expected to become a fully-fledged reality within a decade. All the major giants in the automotive industry are hard pressing their transition from conventional vehicle to autonomous vehicles. The state of Karnataka, for instance, had approximately 205,200 registered taxis higher than Madhya Pradesh 174,900 registered cabs from 2014 to 2015. This presents a great deal of opportunities for autonomous cars and need for technologies. Autonomous cars reduces the accidents rate, stress free parking, saves time, reduces traffic congestion, improve fuel economy etc. It is so sophisticated to the level of easy prediction of physical objects, behavioural elements such as driving speed limits and driving rules between the physical world and its map. Autonomous vehicle have grown to an extent of updating its own information and also based on the cloud, benefitting the systems of all other cars on the network. Machine vision is the most crucial aspect which gives the autonomous vehicles the knowledge of its surrounding. This paper deals with the different approaches of machine vision that helps the vehicle in lane and obstacle detections. Few methods of obstacle detection like Single Object Detection and tracking (SODT) and Multiple Object Detection and tracking (MODT) are compared and contrasted in this paper. Despite the enormous advantages, there are still some challenges of autonomous which needs to be addressed. The challenges that the field will face, especially in relevance with India, along with the suggestion for improvement is also discussed.
\end{abstract}

\section{INTRODUCTION}

Automobile industry is one the fastest growing industry, which makes the transportation of both people and cargo easier, simpler and in less time. The industry not only produces transportation vehicles, but also contributes as a major customers in many other fields such as materials, labor forcers, oil and banking etc., in many countries; automobile field is also one of the major contributors of employment opportunity to many.

In 2017, All over the world, it was predicted that total cars utilized was estimated to 78.6 million vehicles. China and United States of America are the leading giants in the

Revised Version Manuscript Received on August 19, 2019. Engineering, SNS College of Technology, Coimbatore Tamil Nadu. India (e-mail: suriyaprasathgd@ gmail.com)

M.K. Rahgul Poopathi, Assistant Professor, Department of Mechanical Engineering, SNS College of Technology, Coimbatore Tamil Nadu. India (e-mail: rahgulpoopathi@gmail.com)

Swaroop Balaji, UG Student, Department of Mechanical Engineering, SNS College of Technology, Coimbatore Tamil Nadu. India(e-mail: swrpb007@gmail.com)

H. Sridhar, UG Student, Department of Mechanical Engineering, SNS College of Technology, Coimbatore Tamil Nadu. India(e-mail: sridharharidass@gmail.com)

S. Santhosh, UG Student, Department of Mechanical Engineering, SNS College of Technology, Coimbatore Tamil Nadu. India(e-mail: smartsanthoshkumar088@gmail.com)
G.D. Suriya Prasath, UG Student, Department of Mechanical

field of both consumption and production. Few of the major players in the field are Ford, Toyoto, Volkswagen and Daimler. Whereas the major suppliers for automobile industry in terms of spares, technologies etc., are Continental, Robert Bosch, Denzo and Magna.

\section{LITERATURE REVIEW}

Automation of vehicle first surfaced in 1918 and was first exhibited by General Motors in 1939 [1]. Since 1964, many organizations in countries like US, Europe and Japan have developed and searched on the relevant topics along with their individual and joint effort from industries, academia and government [1]. The Defense Advanced Research Projects Agency's (DARPA) Grand Challenges Program in the US in 2004 is considered one of the turning points in the autonomous vehicle field which sky rocketed the research [2]. The American government in 2013 has classified the autonomous vehicles in levels from level 0 to level 5. Level 0 types of vehicles are not automated and are fully controlled by driver. Level 1 car is automated to certain functionalities and level 2 cars are these functionalities combined together. Level 3 cars are self driving cars but with limitations and level 4 are fully fledged autonomous self driving cars. Major problem that semi automatics cars is the compromising state of human error and fatigue associated with it. This would result in major safety concerns [3]. As when talking about the fully autonomous cars, it is required to keep their digital map updated as that is the primary source of information to the car. Any compromise on this to the slightest level might result in loss of property and in worst case scenario, can cause fatalities. [4]. A survey was done by IEEE in 2014 on autonomous vehicles which state that legal liability, policymakers and customer acceptance as the major challenges in mass producing self driven vehicles. Autonomous cars on the whole can improve fuel economy and thus inflicting a positive impact on the environment. [5]. Not only on the fuels, but autonomous vehicles can also bring down the requirement of cars by $15 \%$ which can save $20-30 \%$ of fuel consumption [6]. Considering US, it can reduce the average transportation time of a person to 38 hours per day per year. [7]. It also saves around 1.3 trillion dollars per year which creates new possibilities of job and consumer market and business ideas [9]. According to the World Health Organization in 2013, accidents and death were considered as the eight highest cause of death in the world. 
Autonomous vehicle can also save much travel time as they are well organized and precisely calculated. Autonomous vehicles are claimed to be one of the major users of ITS tools in smart urban transport system [8]. According to a study by Bloomberg in 2017, well over 36 countries are working on autonomous vehicles and 18 countries are looking at the possibilities of long ranged policy making and regulations to accommodate the newly emerging system in its place. Along with benefits to the environment and the economy, self driving vehicles are also said to have a positive impact on humans and their mental and physical health by eliminating driver stress, driver distractions and recklessness that lead to accidents [11]. As with its advantages,it also posses few other challenges as system failure or a sudden breakdown. In both the cases it can cause major problems to cyclist, pedestrians and such in urban areas[12].

\section{AUTONOMOUS VEHICLES}

An autonomous car is a vehicle that can maneuver through an uninformed terrain or route solely by its own, using the information gathered by high end sensing devices. This happens without any human intervention. This once was a dream but thanks to the development of research and development of new technology, autonomous vehicles are no longer a fiction but a reality that is well within the grasp. The main factor that made autonomous vehicle possible in reality is the development of sensing devices and techniques that provides all the necessary information that the vehicle may require to understand and map its surrounding using a smart in-built computer. Various technologies that have contributed majorly for this are lasers and radars, GPS navigation, antilock braking system, cruise control, proximity sensor and so on. With growing demand and advancement in artificial intelligence and machine learning, autonomous vehicle are the most invested field of the day and of the near future.

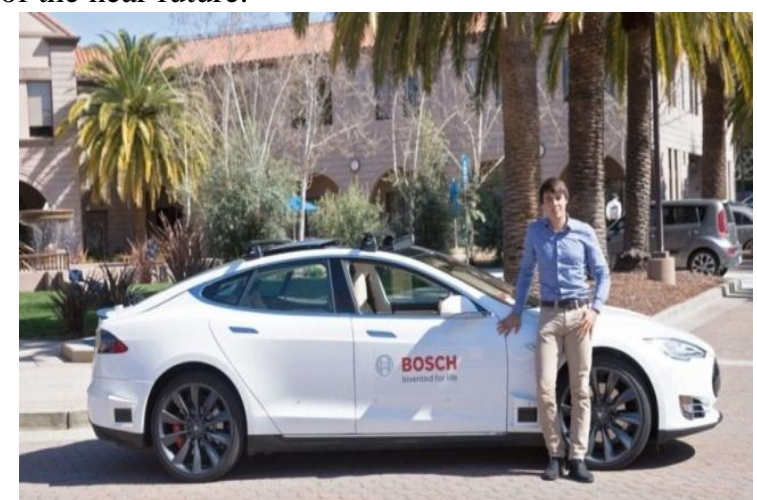

Fig.1: Autonomous Vehicle by Bosch

Without argument, computers are recognized for their speed, accuracy and precision which when incorporated in autonomous vehicles, will replace human errors, emotional compromise of decision while driving and human fatigue. As a result of this accidents can be brought well below the current rate. Not only saving life, but autonomous cars can also play a major role in the ever increasing traffic congestion especially in metros and other major cities. When connected to each other through a network, autonomous cars are much capable of working in a highly synchronized swarm of vehicle. This connected network of cars can positively affect the traffic choking during peak hour in major cities. As odd as it may sound, according to Ohio University autonomous vehicle are capable of reducing co2 emission. This is because of the smartness of the computer that is behind the autonomous.

Autonomous cars use sensors but it brings complications in auto insurance and traffic regulator which the conventional car follows. Autonomous car promises to reduce traffic in road but the constraint here is that introduction of autonomous vehicles may lead to more number of personal cars which increases our time of travel. As autonomous vehicle is a radical change it also demands certain changes in our infrastructure like lane striping, storage place for data collection, installation of charging networks in case of electric cars. Moreover autonomous vehicles at its optimal best will not violate road regulations like over speeding, over staying at parking lots, etc. this reduces notable portion of a state's revenue. Conventionally, insurance is issued based on drivers responsibility but autonomous vehicles throw a major challenge of this as no human controls it. This reduces the magnitude of insurance issual. Autonomous vehicles have high possibility of misuse as far as law enforcement is concerned. For instance narcotics and drugs can be easily transported. The toughest challenge of autonomous vehicles is to maintain social justice and equity.

\section{MACHINE VISION}

Machine vision the technology and method used to provide imaging based on automatic inspections and has applications on process control and robot guidance. Machine vision integrates large number of technologies, software and hardware, integrated systems and expertise. Machine vision integrates new technology and applies them to solve real world problems. It is also used in security and vehicle guidance [8].

Machine vision works in the series of sequential steps. Firstly, the sensor detects if a object is present. If an object passes the sensor, the sensor triggers the camera to capture it and the light source highlights its details. Next the digitizing device called frame Grabber translates the acquired image into digital output which is stored in a computer memory to be processed by software.

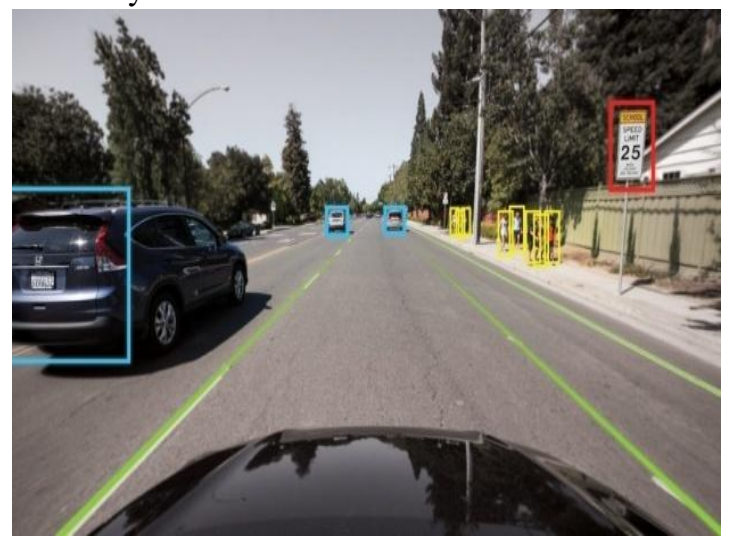

Fig.2: Machine Vision for Object Detection

Published By: Blue Eyes Intelligence Engineering \& Sciences Publication 
Similarly image processing is a step by step process. Here the image is reduced in gradation into simple black and white format. Next the system software analyzes the image to identify the defects and proper components based on predetermined criteria. After analyzing the product while either passes or fails based on machine vision's system findings.

\section{V.MACHINE VISION IN AUTONOMOUS VEHICLES \& RESULTS}

Machine vision plays a very important role in autonomous vehicle for obstacle detection and tracking. An autonomous vehicle which aims to maneuver through an uninformed terrain or road needs all the required information about its surroundings. The information includes the moving and fixed obstacle on the way and the lane that has to be followed. Using machine vision, the vehicle needs to recognize cars, vans, pick-ups, by passers, dividers and so on. The Obstacle Detection and Tracking (ODT) play an important role in achieving this [3].

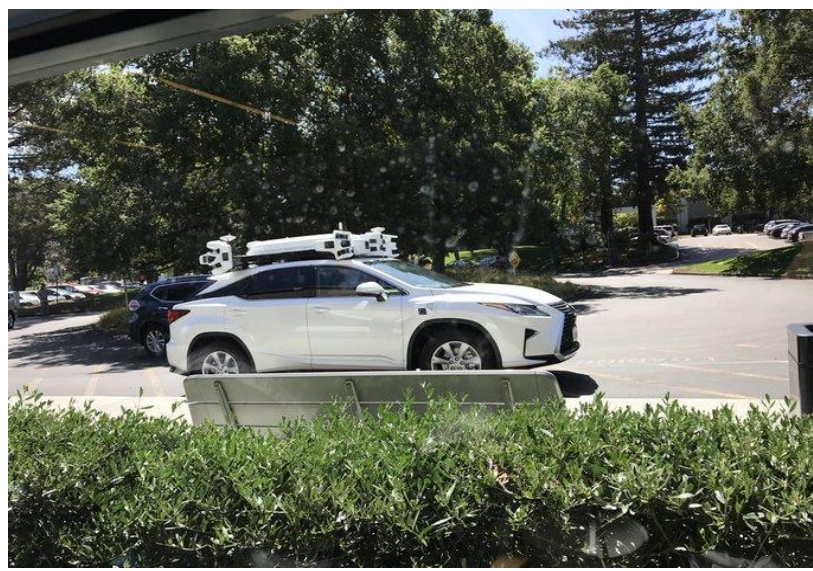

Fig.3: Self-driving car by Apple

An autonomous vehicle uses various input sensors to see its surrounding. Some of the sensors are cameras, radar and lasers. Using these devices the vehicle creates a digital map for itself for navigation. For this to be done, the car needs to do object detection which has two parts as Image classification and image location. Image classification helps the autonomous car to classify the image it got into different objects like car, road sign, van, pedestrians etc., the most used method for this process is convolution neural network. As The second part, image location is to find the exact location and distance of the object detected from the car. Vehicle autonomy is broadly classified into five levels and machine vision plays a very crucial role in level five autonomy [21]. To understand the surrounding the car needs to have a good definition of picture of it's surrounding. The major parameters in machine vision cameras are field of view, frames per second and megapixel. As the field of view needs to extend, so does the megapixel to give image of more clarity. Frames per second plays a very important role in safety as it is directly proportional to the stopping distance of autonomous vehicles [7]. This becomes very crucial when the vehicle tends to move at accelerated field.

\section{CHALLENGES AND PROSPECTS}

Self driving cars, when connected in network can completely stop traffic choking at major level in traffic rich cities. It can avert accident and save to the larger extent of loss of life and property. But the challenge arises that with increased number of vehicle can cause lower traffic efficiency [10]. Though self driving autonomous cars are a boon to the automobile sector, it demands a larger capital investment in infrastructure as current road conditions, especially with reference to a country like India cannot accommodate these vehicles. This puts the government and the managing authorities under a major financial stress. The investment need to be made not only on new lanes and parking but also on technological hardwares and softwares that are related to manage the vehicle [19]. Though autonomous vehicles are under the supervised car of smart computers and devices, cyber security state of today may not be adequate for our country to safe guard the information of the self driving cars [14]. The prospects of autonomous vehicle are many but they also come with their own challenges. Though these challenges post an immediate blockage for implementation of the technology in today's context, the growth of technology posses a promising trend of implementation of self driven cars in the immediate future.

\section{CONCLUSION}

Transportation plays a major role in the development of a country. Especially it can change the tide for a young and dynamic country like India. However, increase in transport also comes with its own risks like traffic congestion, accidents that lead to loss of life and property and so on. When people are put behind the wheels it can cause even intensified problems as not all are equally skilled and cautious in driving. Hence, autonomous vehicles are seen as a need of the hour as it can mitigate and in some cases, eradicate the troubles and problems that arise with human error. Autonomous vehicle can have a very significant positive effect on traffic congestion, mortality rate on accounts of accidents, loss of property, environmental effects and many more. Machine vision helps the autonomous vehicle to understand and move through the undefined lane and it is very promising technology for object detection in tracking for self driving vehicle. Though self driving or autonomous vehicle posses very promising future, it has its own challenges that need to be met before being actually implemented.

\section{REFERENCES}

1. Ernst Dieter Dickmanns, Volker Graefe, "Dynamic Monocular Machine Vision" Machine Vision and Applications, 1998.

2. DARPA, C. http://www.darpa.mil/. [Online] Available at: http://archive.darpa.mil/grandchallenge/overview.html., 2015.

3. Bainbridge, L.,. "Ironies of Automation." Automatica, 19(6), 1983p. 775-779.

4. Knight, W.,. MIT Technology Review. [Online] Available at: http://www.technologyreview.com/featuredstory/520431/driverless -cars-are-further-away-than-you-think/, 2013

5. Luettel, T., Himmelsbach, M. \& Wuensche, H.-J., 2012 "Autonomous Ground Vehicles-Concepts and a Path to the Future.” PROCEEDINGS OF THE IEEE , 100 (Special Issue: SI ), pp. 1831-1839. 
6. Dean Pomerleau And Todd Jochem ,"Rapidly Adapting Machine Vision For Automated Vehicle Steering"1996.

7. Matthew A Turk, "A Vision SystemFor Autonomous Land Vehicle Navigation"1988.

8. Rudolf Gregor et al., "EMS - Vision: A Perceptual System for Autonomous Vehicles" IEEE Transactions on Intelligent Transportation systems , 2002

9. Bartl, M., "The Future of Autonomous Driving - Introducing the Foresight Matrix to Support Strategic Planning "2015

10. M.Maurer et al., "A Compact Vision System For Road Vehicle Guidance” IEEE Proceedings of ICR 1996.

11. Bartl, M., Füller, J., Mühlbacher, H. \& Ernst, H., 2012. “A manager's perspective on virtual customer integration for new product development". Journal of Product Innovation Management, 29(6), pp. 1031-1046.

12. Bertozzi, A. M., Broggib, A. \& Fascioli, A, "Vision-based intelligent vehicles: State of the art and perspectives". Robotics and Autonomous Systems, ., 2000.

13. Broggi, A. et al.,. "Extensive Tests of Autonomous Driving Technologies." IEEE TRANSACTIONS ON INTELLIGENT TRANSPORTATION SYSTEMS, 14(3) 2013.

14. Browning, J. G.,. "Emerging Technology and Its Impact on Automotive Litigation.” Defense Counsel Journal, 81(1), pp. 83-90. 2014.

15. Chubin, D. E. \& Hackett , . E. J.,. Peerless science: peer review and US science policy, New York: Albany, State University of New York.. 1990.

16. Dahlander, L. \& Gann, D. M.,. How open is innovation?. Research Policy, 39(6), pp. 699-709, 2010.

17. Ross, P. E.,. "Robot, you can drive my car; Autonomous driving will push humans into the passenger seat”. IEEE SPECTRUM , 2014.

18. Morgan Stanley, Autonomous Cars, Self-Driving the New Auto Industry Paradigm, 2013

19. Payre, W., Cestac, J. \& Delhomme, P., "Intention to use a fully automated car; Attitudes and a priori acceptability." Transportation Research Part F: Traffic Psychology and Behaviour, Band 27, Part B, pp. 252-263. 2014

20. Waytz, A., Heafner, J. \& Epley, N.,. "The mind in the machine; Anthropomorphism increases trust in an autonomous vehicle." Journal of Experimental Social Psychology, Band 52, pp. 113-117. 2014.

21. Weyer, J., Fink, R. D. \& Adelt, F., "Human-machine cooperation in smart cars. An empirical investigation of the loss-of-control thesis." Safety Science, Band 72, pp. 199-208, 2015.

22. Wijesoma, W. S., Kodagoda, K. R. \& Balasuriya,. "A. P. RoadBoundary Detection and Tracking Using Ladar Sensing." IEEE Transactions on Robotics \& Automation, 20(3), pp. p456-464. 9p 2004.

23. World Health Organization. Global Status Report on Road Safety, 2013 